\title{
APORTES DE LA ECONOMÍA FEMINISTA PARA EL ANÁLISIS DEL CAPITALISMO CONTEMPORÁNEO Cristina Cielo ${ }^{1}$, Héctor Fabio Bermúdez ${ }^{2}$, Andrea Almeida Guerrero ${ }^{3}$ y Mariela Moya ${ }^{4}$
}

\section{Resumen/Abstract}

Desde hace décadas, feministas economistas han demostrado que las diferenciaciones y jerarquizaciones entre hombres y mujeres son fundamentales para la organización de las sociedades capitalistas. En este artículo, vemos que los análisis feministas cobran una nueva fuerza y relevancia en el contexto de los rasgos y mecanismos del capitalismo contemporáneo. Enfatizamos tres aportes principales de sus estudios. Primero, con su señalamiento de la invisibilización del trabajo reproductivo y emocional, perspectivas de la economía feminista ayudan entender la creciente importancia de las formas cognitivos y relacionales del trabajo, cada vez más importantes en relación con el ámbito productivo formal. Las transformaciones en el trabajo subyacen la redistribución de riesgo en la economía postfordista, lo cual nos lleva al segundo aporte fundamental de estudios feministas de la economía. Estos estudios arrojan luz sobre las vivencias materiales y subjetivas de poblaciones precarizadas, cuya inseguridad económica refleja su carga desigual de los riesgos característicos de la neoliberalización y financiarización de la economía. Finalmente, vemos cómo estas dinámicas aportan a las nuevas formas de la producción y la transferencia de valor, deteniéndonos en el énfasis de estudios feministas en las desigualdades experimentadas desde los lugares externalizados y expropiadas por la acumulación de capital.

Palabras clave: Economía feminista, trabajo relacional, distribución de riesgo, transferencia de valor.

1 Estadounidense. Facultad Latinoamericana de Ciencias Sociales (FLACSO-Ecuador). E-mail: mccielo@flacso.edu.ec

2 Colombiano. Facultad Latinoamericana de Ciencias Sociales (FLACSO-Ecuador). E-mail: hectorfabio6@gmail.com

3 Ecuatoriana. Facultad Latinoamericana de Ciencias Sociales (FLACSO-Ecuador). E-mail: andre_nth1011@ hotmail.com

4 Boliviana. Facultad Latinoamericana de Ciencias Sociales (FLACSO-Ecuador). E-mail: killaymar256@gmail.com 


\section{CONTRIBUTIONS OF FEMINIST ECONOMICS TO OUR ANALYSIS OF CONTEMPORARY CAPITALISM}

For decades, feminist economists have shown that differentiations and hierarchies between men and women are central to the organization of capitalist societies. In this article, we review the renewed forms and relevance of feminist analyses in the context of contemporary capitalism's particular features and mechanisms, highlighting three principal contributions of their insights. First, given their demonstration of the invisibility of reproductive and emotional work, feminist economics perspectives help us to understand the growing importance of cognitive and relational forms of work, which are increasingly important in relation to the formal productive sector. Transformations in labor underlie the redistribution of risk in the post-Fordism economies, which leads us to the second fundamental contribution of feminist economic studies. These studies shed light on the material and subjective experiences of precarious populations, whose economic insecurity reflects the unequal distribution of risk that is characteristic with the neo-liberalization and financializing of the economy. Finally, we see how these dynamics contribute to new forms of production and transfer of value, emphasizing feminist studies of inequalities experienced in the externalized and expropriated sites of accumulation of capital.

Keywords: Feminist economics, relational labor, distribution of risk, transfer of value 
Un enfoque feminista a la economía va más allá de visibilizar las relaciones desiguales de género en nuestras relaciones laborales y socio-económicas. Desde la década de los setenta, feministas economistas (Dalla Costa y James 1971, Scott y Tilly 1978, Mies 1986) han argumentado que las diferenciaciones y jerarquizaciones entre hombres y mujeres son fundamentales para la organización de las sociedades capitalistas. Trabajos de la economía feminista cobran nueva fuerza y relevancia en el contexto de los rasgos y mecanismos contemporáneos de la acumulación del capital, particularmente en el contexto de la transformación en las formas de trabajo, nuevos riesgos económicos y mecanismos de transferencias de valor. En este artículo, vemos cómo nuestros estudios de procesos y desigualdades económicos actuales se pueden mejor precisar y profundizar tomando en cuenta los aportes conceptuales y metodológicos de los acercamientos feministas.

Empezamos el artículo examinando los aportes de la economía feminista que nos ayudan a comprender las nuevas formas de trabajo. Desde sus primeros estudios, analistas feministas han señalado la invisibilización del trabajo reproductivo y emocional (Pérez Orozco 2014, Carrasco 2003, Hoschild 1985). Estos análisis dan pistas para entender la creciente importancia de las formas del trabajo cognitivo y afectivo (Miguez 2008;), y la relación de estas con el trabajo del ámbito productivo formal (Vercellone 2013). En el segundo apartado, vemos cómo estas transformaciones en el trabajo subyacen la redistribución de riesgo en la economía postfordista. Estudios sobre la precarización del trabajo y de las identidades laborales de las mujeres (Todaro y Yañéz 2004, Salas y Pérez Sainz 1997) arrojan luz sobre las dificultades que experimentan las poblaciones más vulnerables, cuya inseguridad económica refleja su carga desigual de los riesgos característicos de la neoliberalización y financiarización de la economía (Lipuma y Lee 2004, Christophers 2015, Terranova 2015).

En el apartado tres vemos cómo estas dinámicas aportan finalmente a las nuevas formas de la producción y la expropiación de valor (Fumagalli 2010, Harvey 2008). Estudios feministas (Galcerán 2006, Federici 2004) que se enfocan en la transferencia de valor desde lugares externalizados de la acumulación de capital nos proveen de herramientas para analizar estas dinámicas en sus múltiples escalas. A lo largo del texto, aterrizamos nuestras discusiones a través del caso del Sistema de Venta Directa, en el cual la ausencia de una relación laboral de dependencia entre empresarios y vendedoras directas repercuta en un frágil estatuto de trabajo para éstas. Esta 
condición permite tanto la precarización de sus condiciones de vida como la transferencia del valor hacia los trabajadores formales de las empresas. En cada apartado, mostramos la manera en que el análisis de estas dinámicas desde la economía feminista es clave para nuestra comprensión de las estructuraciones económicas contemporáneas.

\section{Para la comprensión de las nuevas formas de trabajo}

Uno de los principales intereses de la economía feminista ha sido la relación histórica y persistente entre el trabajo doméstico y la acumulación capitalista. Desde hace décadas, analistas feministas han argumentado con vehemencia que el trabajo doméstico reproduce la fuerza de trabajo y sostiene al trabajo denominado "productivo" (Himmelweit 2011, Dalla Costa y James 1971, Picchio 1994). Un referente básico en estas discusiones es el trabajo de Silvia Federici $(2004,2013)$, que da cuenta de un proceso histórico complejo en el que la acumulación del capital y la racionalización de la reproducción social desvaloriza, oculta y niega una serie de actividades sociales que garantizan las condiciones para la reproducción material, simbólica y social de la vida. Para Federici (2004), la profundización de la división del trabajo, el confinamiento de las mujeres al ámbito reproductivo y la desvalorización de su trabajo fueron parte del proceso propiamente violento y destructor que acompañó a la instauración del capitalismo como sistema mundial. Estos tipos de miradas ofrecen un análisis más amplio del papel de las mujeres en el capitalismo y demuestra el protagonismo de larga data de las mujeres en la constitución de distintos espacios económicos.

Los estudios sobre la participación histórica de las mujeres en el trabajo productivo señalan la artificialidad de la escisión producción-reproducción. Scott (1993) expone claramente que, a lo largo de su historia laboral, las mujeres han "compaginado" los trabajos considerados productivos y aquellos que permiten la reproducción de sus familias y comunidades. Lo singular del periodo industrial fue la organización de la productividad económica alrededor del trabajo en las fábricas, lo cual despojó a la familia de sus funciones productivas (Scott y Tilly 1978). Al mismo tiempo, esto profundizó la subvaloración e invisibilización de los trabajos reproductivos y de cuidado que tuvieron lugar fuera de la producción manufacturera, siendo esta denuncia sobre la invisibilización de ciertas formas de trabajo uno de los aportes más importantes de estas autoras. Múltiples formas de trabajo desvalorizadas como no-productivas mantienen una relación simbiótica con el capital y que se apropian para la acumulación de excedentes (Pérez Orozco 2014). 
En este sentido, el análisis de Cristina Carrasco (2003) es importante por cuanto identifica la existencia de "la poderosa 'mano invisible' de la vida cotidiana" para señalar el ocultamiento de trabajos cotidianos cuya importancia se desestima. Este es sobre todo el caso de aquellos trabajos que implican relaciones afectivo-sociales y que generan un complejo tejido de relaciones humanas sobre las que se sustenta la sociedad en su conjunto. Carrasco ofrece una caracterización de este tipo de trabajos reproductivos como aquellos que se realizan "[...] tanto en el hogar y fuera de él, en el barrio y desde el puesto de trabajo remunerado, que crean redes familiares y sociales, que ofrecen apoyo y seguridad personal y que permiten la socialización y el desarrollo de las personas" (Ibíd.: 17).

De manera paralela, varios economistas políticos (Fumagalli2010, Morini 2014) han señalado la centralidad del trabajo relacional en lo que identifican como la nueva fase del capitalismo que emerge desde la crisis del fordismo. Estos teóricos argumentan que las transformaciones en las formas del trabajo han significado un conflicto creciente entre "el carácter social de la producción y el carácter privado de la apropiación” por el capital (Vercellone 2013: 3). Pablo Miguez (2008) señala que en las transformaciones de la relación capitaltrabajo, "el actor fundamental del proceso de producción" es el saber social general, el general intellect del que hablaba Marx (1993). Este implica "una cooperación social más amplia y heterogénea que la específica al campo de trabajo. Se trata de facultades afectivas, cognitivas, donde participan todos los sujetos" (Miguez 2008: 13-14). Negri caracteriza la nueva forma del trabajo, "que no sólo crea bienes inmateriales, sino también relaciones y, en última instancia, la propia vida social” (Negri 2004 en Miguez 2008:17).

Las economistas feministas, por su parte, advierten que estas formas de trabajo no son nuevas. Lo importante es comprender su nuevo rol y las formas actuales en que se apropia de este trabajo invisibilizado, cuya realización es imprescindible para la reproducción de lo social, en sus dimensiones simbólicas, afectivas y políticas, además de materiales. En todos los servicios, especialmente los que implican una relación cara a cara, los trabajadores despliegan formas de gestión emocional y afectiva. En la clásica investigación sobre la labor emocional, Arlie Hochschild (1983) comprobó el gran trabajo que despliegan las azafatas de una aerolínea que involucra el control de sus emociones. Podemos ver estos elementos en el estudio de ocupaciones como el Sistema de Venta Directa (en adelante SVD), que se sitúan en las fronteras del trabajo físico y emocional consumo y vida social. El componente emocional 
impreso en la venta directa y la instrumentalización de las redes familiares son puestas al servicio de la acumulación capitalista y aprovechadas por los empresarios.

La venta directa - en la que mujeres venden productos de Avon, Yanbal, Ésika, etc. a sus amigas y familiares - ha sido comúnmente definida como una modalidad en la cual los empresarios eliminan la fase de intermediación de los circuitos productivos. Las vendedoras, que son la fuerza de trabajo de las empresas, revenden los productos a cambio de una remuneración basada en un porcentaje por facturación. Un elemento central de estas modalidades es el desempeño de trabajo emocional, el cual requiere de inversión significante de tiempo y esfuerzos, que sin embargo es usufructuado por las empresas, invisibilizado y no pagado. Las empresas de SVD fomentan una organización del trabajo que horizontaliza las relaciones sociales (Miyata y Suzuki 2011), instrumentalizando redes sociales y familiares (Ramírez y Rúa 2008). De esta forma se tejen y aprovechan redes sociales en las que se involucran sentimientos, confianzas, solidaridades y alianzas comunitarias.

El análisis concreto de casos como el del SVD permite identificar las formas de la apropiación del trabajo relacional, simbólico e inmaterial por parte del capitalismo. De hecho, feministas han desarrollado de manera crítica la propuesta conceptual del trabajo inmaterial de Hardt y Negri 2000, 2004, 2011). Si bien estos autores señalan que esta forma de trabajo caracteriza los nuevos antagonismos capital-trabajo, feministas demuestran que su análisis minimiza las diferenciaciones y relaciones desiguales entre distintos grupos de trabajadores. La forma abstracta en la que Hardt y Negri conceptualizan el trabajo afectivo, como parte del trabajo inmaterial, mistifica el carácter corporal y divisiva de buena parte del trabajo de reproducción social (Federici 2013:192), dando paso a poca comprensión de las jerarquías e inequidades entre trabajadores y las luchas históricas que diversos grupos han mantenido para visibilizar y reivindicar su marginalización.

En este apartado hemos visto que la problematización del trabajo invisibilizado de las mujeres señala maneras de estudiar el papel de todos los trabajos que no se consideran productivos. A partir de su enfoque en la reproducción y el trabajo emocional, los aportes de la economía feminista insisten en la importancia de comprender el rol fundamental y desvalorizado que juegan los trabajos no-formales, colectivos y sociales, para la productividad económica. A su vez, dan cuenta de la pluralidad desigual de estas formas de trabajo y por consiguiente de la constitución heterogénea del capitalismo 
(Quiroga y Gómez 2013). El rastreo del pensamiento de la economía feminista demuestra que la instalación de las nociones de producción, trabajo y economía se consolida precisamente sobre el ocultamiento de la productividad fundamental de los trabajos reproductivos y emocionales. Veremos en el siguiente apartado las maneras en que las vendedoras responden a esta desvalorización de su trabajo a través de la construcción de su identidad laboral, y la forma en que estos procesos precisan los impactos desiguales de la inestabilidad económica.

\section{Para la comprensión de la redistribución desigual del riesgo}

Los fuertes procesos de degradación de las condiciones y formas del trabajo que sufren las y los trabajadores en la actualidad ya venían incrementándose desde el último tercio del siglo con la crisis del modelo fordista, el debilitamiento de los estados benefactores y la instauración de un nuevo espíritu del capitalismo (Boltanski y Chiapello 2002) sustentado en procesos de flexibilización. En las postrimerías del siglo XX, Donna Haraway (1995) ya advertía sobre la feminización de la pobreza, término que lejos de referirse exclusivamente a la situación de las mujeres, da cuenta de la manera en que el sustento de la economía neoliberal usufructúa cada vez más del trabajo invisibilizado de reproducción señalado en el apartado anterior. Estas transformaciones han influido en la modificación de los contenidos del trabajo: como vimos arriba, los trabajos se orientaron al despliegue de capacidades y habilidades comunicativas y relacionales. Varios autores han relacionado estos procesos con el fin del consumo de masas (Harvey 2008) y el desarrollo del trabajo inmaterial (Lazzarato y Negri 2001).

A la vez, las condiciones de trabajo también se han visto transformadas, viéndose caracterizadas por mayores niveles de cualificación, polivalencia y ritmos cambiantes, empleos inestables, ausencia de seguridad social, subempleo, individualización de las relaciones salariales e ingresos precarios (Todaro y Yáñez 2004). Los análisis de la economía política de este periodo han advertido que la precarización sufrida por los trabajadores se debe a la redistribución del riesgo en la economía contemporánea. En este apartado vemos la manera en que estos análisis se pueden enriquecer por los estudios de las subjetivaciones y la precarización que han experimentado las mujeres. Estudios feministas arrojan luz sobre las diferenciaciones desiguales que experimentan las poblaciones más vulnerables, y cómo se les carga las inseguridades económicas a través de procesos materiales y subjetivos. 
El caso de las líderes de Avon aludido arriba resulta emblemático. Ellas, sin haber pactado una relación de dependencia laboral con la empresa, se encargan de todas las dificultades y los riesgos de su venta: tienen que presionar a sus representantes, llamarlas incansablemente a través de una línea telefónica pagada por ellas mismas, asegurar que realicen oportunamente sus pedidos de campaña, etc. El trabajo de estas vendedoras, como cualquier otra categoría analítica está en permanente disputa. En ese sentido, su abordaje no solo requiere una consideración de las condiciones materiales que lo constituyen, sino también de los significados que le atribuyen los distintos actores e instancias de poder que participan en su delimitación. En este sentido, debemos entender el trabajo precarizado de las vendedoras en sus distintas dimensiones: por las denominaciones provenientes de los organismos estatales a través de las cuales se etiqueta a conjuntos de trabajadoras y trabajadores (o se los excluye de tales denominaciones) mediante el impulso y aplicación de políticas públicas; y por las significaciones que tienen las trabajadoras sobre sus actividades, además de las formas de nombrarse a sí mismas.

Es esta última dimensión subjetiva la que se pone en juego cuando las empresas les insisten a las vendedoras: "eres la jefa de tu propio negocio". Ellas, por su lado, construyen socialmente su ocupación a través de procesos de cualificación social, capacitándose para enseñar y vender los productos que aparecen en las imágenes de sus catálogos, desarrollando destrezas emocionales para mejor establecer confianza con las clientas. Las vendedoras más experimentadas saben identificar la calidad y los atributos de los productos y también a los buenos y malos clientes. En este sentido, se elaboran identidades laborales alrededor de esta cualificación y de la gestión de los afectos. Además, la organización del trabajo en redes familiares y comunitarias permite el establecimiento de alianzas entre vendedoras, el intercambio de favores, y en suma la constitución de nichos de solidaridad que trascienden el simple interés mercantilista. En esa medida, algunas de ellas perciben sus actividades como una oportunidad y como identidad.

En el despliegue de una serie de saberes, destrezas y estrategias orientadas a fines lucrativos, las vendedoras pretenden incrementar sus ganancias tratando de reducir al mínimo el riesgo crediticio. Por riesgo crediticio entendemos el hecho de que las ventas se hacen a crédito, siendo las vendedoras quienes asumen las pérdidas económicas causadas por los clientes morosos. Cuando las vendedoras se endeudan por estos clientes morosos, aparece el argumento de las buenas maneras de administrar el negocio propio. El lema de los SVD mencionado arriba que les pone a las mujeres a cargo de sus propias empresas 
y negocios debería ser completado con el verdadero interés de los empresarios: "ya que eres la jefa de tu propio negocio asume tú la cobertura social del riesgo crediticio". Observamos varios puntos importantes en el orden del riesgo. Primero, la posibilidad de redistribuir el riesgo a las vendedoras se construye sobre la desvalorización de su trabajo relacional y emocional. Segundo, la manera en que estas mujeres se responsabilizan del riesgo crediticio también depende de las identidades laborales y subjetividades emprendedoras que ellas construyen. Finalmente, vemos que la organización de la empresa y el poco reconocimiento legal del trabajo de las vendedoras legitima este orden del riesgo en el que las más vulnerables de la empresa cargan el riesgo mayor del negocio.

Estos tres puntos añaden elementos importantes para un análisis de la financiarización, que se enfocan en la redistribución de los riesgos (Lazarrato 2013, Gago 2014). La crisis de hipotecarias subprime en los Estados Unidos demostró que las ganancias formidables por herramientas financieras como las Obligaciones de Deuda Colaterizadas se debían a una redistribución innovadora del riesgo. La manera en que funcionan estos derivados recaudando un conjunto de bonos y activos diversificados para transar sus valores en tranchas diversas según posibilidades de capital y riesgo - termina fortaleciendo aún más las desigualdades. Cuanto mayor capital se tiene para invertir, menor es el riesgo que debe asumir. Lipuma y Lee (2004) explican cómo funciona la objetivación, circulación y redistribución del riesgo a nivel global, a través de los derivados financieros: las estrategias de cobertura de corporaciones del capital especulativo pueden llevar a la devaluación de monedas, a la inflación y al agotamiento de las reservas de divisas de países periféricos, cuyos ciudadanos más precarizados experimentan los impactos más fuertes de estos procesos, en términos de aumento desempleo y de precios de la canasta familiar.

Los trabajos de la economía feminista que hemos revisado complementan estas reflexiones, enfatizando los impactos distintos de este orden del riesgo en el nuevo capitalismo para las poblaciones diferenciadas históricamente y de manera desigual, por género, clase, raza y etnia y nación. Por lo tanto, la distribución de los riesgos pasa también por la constitución y definición de identidades y sujetos. Verónica Gago precisa las maneras en que las lógicas del capital financiero se articulan con la cotidianidad y las subjetividades urbano-populares: "Lo que estas finanzas leen e intentan capturar es la dinámica de sujetos ligados a la estructuración de nuevas formas laborales, emprendedoras, autogestoras que surgen en los sectores pobres en paralelo a su 
condena como poblaciones sobrantes o excedentes" (2014: 212). Estos sujetos, como nos insisten los aportes feministas que hemos revisado, se constituyen sobre la invisibilización y jerarquización de tipos particulares de trabajos y de poblaciones.

Los estudios de la economía feminista, además, han aportado en la consideración de las distinciones y valorizaciones de diferentes espacios: espacios públicos y privados, la calles y la casa, lugares urbanos y rurales, centros comerciales, mercados populares, etc. Estos diversos espacios son atravesados no solo por relaciones de género, sino también por otros tipos de diferencias ancladas en la clase, la etnia, que nos permite captar la complejidad de las relaciones gestadas en el desempeño de múltiples trabajos, los mismos que rompen con la dicotomía de productivo-reproductivo y que se desenvuelven como una continuidad cuya finalidad es el sostenimiento de la vida humana. Esta mirada exige replantear la problemática de las diferenciaciones desiguales necesarias y el orden de riesgo que las acompaña en función de la acumulación de capital. Asimismo, se pone de primer plano las subjetivaciones y la precariedad de las poblaciones más vulnerables, acompañadas por la desvalorización e informalización de su trabajo, aspectos que les acarrean inseguridades económicas inequitativas.

Para comprender las nuevas formas de producción de valor y su explotación

En este apartado exponemos cómo los debates y las reivindicaciones en relación al trabajo doméstico y al trabajo de cuidado desafían de manera contundente los planteamientos economicistas que externalizan la reproducción material y social. Aportan, por lo tanto, elementos en el análisis más general de la transferencia de valor desde lugares externalizados a los procesos de acumulación de capital. Los análisis actuales de esta captura de valor por los procesos acumulativos identifican la producción de valor en ámbitos no considerados productivos - como son los del consumo y de los recursos naturales - y los mecanismos para su apropiación y explotación. Por su parte, los trabajos de las feministas examinan y visibilizan el valor de las actividades consideradas reproductivas para el conjunto de la economía (Himmelweit 2011), para especificar la relación entre este ámbito externalizado y la producción capitalista. Nos ofrecen una aproximación conceptual a la transferencia de valor que permite vincular las múltiples escalas de apropiación y acumulación. 
Las economistas feministas han demostrado que las economías familiares están atravesadas por relaciones desiguales de género, y en ellas se realizan distintos tipos de trabajos que sustentan otros espacios. Es decir, la distribución desigual del trabajo genera excedentes que se acumulan a través de las formas directas e indirectas de apropiación. Podemos extender este punto para entender la expropiación y transferencia de valor en el análisis más allá del caso de estas familias en particular. Por ejemplo, se puede pensar en la distribución dispar de la tierra y recursos naturales en el sector rural o los espacios desiguales urbanos, a partir de los cuales se generan múltiples estrategias de supervivencia que incluyen el trabajo precarizado que transfiere valor a través de relaciones asimétricas de intercambio. El análisis de Carrión y Herrera sobre la economía familiar campesina señala este punto: "el mercado como mecanismo de distribución de recursos e ingresos beneficia siempre a quien posee más capital, generando transferencia de valor de unos sectores a otros" (2012: 112). Estas relaciones de intercambio se encuentran insertas en mercados fuertemente controlados por complejos industriales y financieros, donde pocas empresas están en capacidad de controlar la mano de obra precarizada. Esto se observará empíricamente más adelante cuando regresemos al caso de las trabajadoras del sistema de venta directa.

Desde esta perspectiva, evidenciamos la manera en que las llamadas economías familiares, constituidas como lugares externalizados de la producción y la acumulación del capital, sin embargo, contribuyen a la creación y transferencia de valor hacia los circuitos mercantiles de la economía global. Se convierten estas economías no-reconocidas en espacios de sustento para la pervivencia de estos circuitos. Este fenómeno toma distintas formas y los análisis de las economías familiares señalan la existencia de "sistemas económicos que no responden solamente a los principios de la economía empresarial capitalista" (Vásconez 2012, 106), que se apoyan como hemos visto en redes familiares para la producción de bienes y servicios.

El examen de las formas en las que las familias sustentan las crisis cíclicas del capitalismo también nos permite vislumbrar la economía política que vincula el trabajo de cuidado con las estructuras desiguales globales. Las cadenas globales de migraciones y de trabajo doméstico, cuya informalidad en muchas ocasiones conlleva su mayor explotación, han permitido sortear las crisis de los cuidados en las economías centrales. En este sentido, vemos de manera paralela que la existencia de "otras economías" - sistemas que responden a formas cooperativas, solidarias, relaciones comunitarias, familiares, asociativas y domésticas, basadas en relaciones de reciprocidad, 
retribución y recirculación de recursos - ha servido de soporte a la economía monetarizada, especialmente en la periferia.

Alison Vásconez señala que en la región latinoamericana la economía se presenta como un continuum entre producción-reproducción, los comportamientos económicos son diversos y múltiples y "se mueven en la economía paralela, las economías de subsistencia, el mercado, el ámbito doméstico, el de circulación no monetaria, de forma simultánea y a veces a cargo de los mismos agentes" (Vásconez 2012: 113). Es así que en las economías domésticas se producen bienes y servicios de distintas características: alimentación, cuidado directo de personas, bienes comercializables, todos ellos sobre la base de lazos familiares, comunitarios, redes de amistad, entre otras, que producen valores de uso concretos y que son generadores de bienestar cotidiano, pero que "no están totalmente reflejados en el mercado y en el precio" (114). Los problemas de valorización de capital del mercado y del precio han sido el enfoque de los análisis de las crisis económicas y financieras (Brenner 2009), pero las perspectivas feministas demuestran que las crisis se tienen que entender de manera multidimensional (Pérez Orozco 2014), en términos de la precarización del trabajo y de la vida, el deterioro medioambiental, la crisis de reproducción en el sur global, todos relacionados, eso sí, con la caída financiera de las economías centrales.

Cuando la valorización del capital estalla, el Estado aplica políticas de ajuste, y ante los efectos de la crisis, los hogares sufren un reacomodo en sus condiciones de vida. Esto sucede a través de la intensificación del trabajo no remunerado en los hogares, trabajo doméstico y de cuidado asumido por las mujeres, la emergencia de formas de auto-empleo, de emprendimientos y de las llamadas "economías de rebusque" (Pérez 2014: 144). Son, entonces, las estrategias de supervivencia generadas desde las familias las que actúan como un amortiguador ante las crisis de acumulación de capital, sustituyendo los bienes y servicios producidos en los hogares por aquellos ya difícilmente accesibles en el mercado, como podría ser el cuidado de los niños. El sustento de la economía formal, particularmente en periodos de crisis, ha sido sostenido en circuitos externalizados y mediante trabajo no remunerado.

Muy ligado a este problema se encuentra la "crisis de los cuidados" al que nos referimos anteriormente. Vásconez define esta crisis como "la limitada y decreciente oferta de trabajo doméstico no remunerado que sostiene los hogares en muchos países, debido ante todo a que la dependencia se eleva y las mujeres (proveedoras históricas de cuidados) tienen menos tiempo para 
este trabajo" (2012: 86). Este problema se ancla en la realidad de países europeos cuya población adulta mayor dependiente se incrementa, mientras que la oferta de trabajo que suple estas necesidades es limitada. Para Vásconez y otras autoras (Anderson 2007), las cadenas globales de cuidado en las que se insertan mujeres diversas es un mecanismo fundamental a través de lo cual ellas entran a la globalización. De esta manera, se recrean formas de interdependencia donde existen flujos de personas y recursos portadores de valor que se trasladan de unas regiones a otras, lo cual explica "la transferencia de valores monetarios y de trabajo desde la región latinoamericana... De hecho, bajo la interdependencia se debería hablar del 'intercambio desigual' de cuidados" (Vásconez 2012: 109). Estos procesos, además, se deben situar en el contexto de una repartición inequitativa y racializada (Vega 2015) de estos tipos de trabajo.

Estas perspectivas no solo nos instan considerar que la posibilidad de reproducción capitalista descansa sobre las economías no contabilizadas como tal (incluyendo a la economía familiar), sino que también nos exige atender a la división de trabajo - jerarquizada y diferenciada por sexo, raza, clase y nación (Quijano 2000) - que tiene lugar al interior de estas economías otras. Espacios domésticos, informales y asociativos no son unidades homogéneas y armónicas, sino que son espacios de cooperación y conflicto, en las que si bien hay una "cierta gestión común del bienestar... también son escenario de conflicto, de relaciones de poder, de distribución desigual e injusta de lo que se hace, quién lo hace, qué recibe a cambio, cómo se valora lo que cada quien aporta y necesita" (Pérez 2014: 163). Estas perspectivas de la economía feminista, entonces, demuestran la importancia tanto de reflexionar sobre los procesos de producción y reproducción, sus rupturas y continuidades y las cargas de trabajo y relaciones asimétricas entre los miembros de tales espacios relacionales, como de comprender la relación entre estas dinámicas y las estructuraciones macro de la economía global.

Con estas percepciones, los aportes de las feministas son importantes interlocutores con otros análisis que visibilizan formas en que la externalización de la producción de valor es central a las nuevas dinámicas del capital. El trabajo de David Harvey (2004), por ejemplo, señala las maneras en que la desposesión de los cercamientos o de los países coloniales - es decir, de lugares que se externalizaron de la producción - no solamente existieron como parte de los procesos de acumulación originaria instaurados con el capitalismo, sino que son procesos continuos de despojo que acompañan el sostenimiento del mismo sistema. Por otra parte, los analistas del capital financiero enfatizan la 
producción de valor en la sociabilidad misma. Marazzi (2015), por ejemplo, señala el trabajo que cada uno hacemos cotidianamente como "prosumidores", produciendo parte de lo que consumimos. En nuestro uso de Google o nuestro ensamblaje de los muebles que compramos en tiendas como IKEA, "hay una transferencia permanente de trabajo no remunerado del consumidor" (Marazzi 2015: 48).

Los acercamientos de la economía feminista aportan a estos análisis contemporáneos de la producción y transferencia de valor, a partir de su insistencia en situar y corporalizar las dinámicas de estas apropiaciones en la acumulación del capital. Situar y corporalizar nuestros análisis de la economía contemporánea se hace necesario para precisar las relaciones entre la organización desigual de estructuras capitalistas y las inequidades vividas por las poblaciones específicas. Ahora bien, para ilustrar los argumentos expuestos retomamos el caso de los sistemas de venta directa, donde se evidencia cómo las empresas se sirven de formas de trabajo realizado por las mujeres vendedoras.

Es precisamente la división sexual del trabajo, la invisibilización del trabajo afectivo y reproductivo de estas "amas de casa emprendedoras", además de la constitución de sus subjetividades dependientes, que permite la transferencia de valor a los dueños e inversionistas de las empresas de venta directa. La base de la pirámide en los organigramas empresariales está compuesta por quienes no gozan de una relación de dependencia laboral. Son las "clientas" entre comillas quienes sostienen al sistema y sus jugosos ingresos. En el año 2014, la industria global movió \$182.123 millones de dólares, según cifras de la Federación Global de Asociaciones de Venta Directa. Aquí se entiende como se sostiene la economía formal y global mediante formas precarias de trabajo y no únicamente en situaciones de crisis. Es así que se vinculan desigualdades cotidianas, identidades y subjetividades y trabajo emocional, social y asociativo a amplios procesos económicos marcados por las transformaciones del sistema capitalista desde finales del siglo XX.

Estos procesos, así como las distintas maneras en que las economías familiares subsidian al sistema de acumulación de capital cuestionan clivajes como el de capital-trabajo, y amplían conceptos analíticos como la división del trabajo y la organización social del trabajo no reconocido (Rodríguez 2014: 34). En síntesis, "el proceso de valorización tiene una serie de costes (como el coste de reproducir la mano de obra en su totalidad, de reponer aquello que se desgasta) que el capital no puede asumir, sino que debe derivar hacia fuera de su propio circuito" (Pérez 2014, 112). Las perspectivas de la economía 
feminista visibilizan la generación externalizada de valores que sostienen la economía capitalista, que por lo tanto descansa en gran parte sobre relaciones no mercantiles. Llaman la atención a la necesidad de analizar la transferencia de valor a partir de los distintos tipos de trabajos posibles para y realizados por grupos específicas de personas. Nos invitan a pensar en las maneras en que estas divisiones y distinciones no sólo crean desigualdades, sino que son aprovechadas precisamente para sostener la apropiación del valor que subyace a la continuidad de las formas de organización y acumulación del capital.

\section{Conclusiones}

Los aportes de la economía feminista al análisis de formas contemporáneas del capitalismo no son solamente conceptuales, sino también metodológicos. Una metodología desde la economía feminista nos invita a observar los trabajos "invisibles" y los sujetos que los realizan. Se trasciende la mirada economicista limitada al ámbito "productivo" es decir en la producción de mercancías, considerando también los múltiples intercambios de bienes, flujos de afectos y relaciones que atraviesan la cotidianidad en las familias, comunidades, lugares de trabajo, empresas, centros de enseñanza, entre otros espacios. En este sentido, las categorías analíticas que proporciona la economía feminista son importantes para el rastreo mundos de trabajo que son invisibilizados, junto a sus actores y sus experiencias, y que dan cuenta de un proceso social e histórico de encuentros e interacciones con las lógicas del capitalismo.

En este sentido, los estudios de la economía feminista ayudan mejor entender las transformaciones en el trabajo de la economía actual a través de su enfoque en la invisibilización del trabajo reproductivo y emocional. Esta visibilización, a su vez, permite registrar la desigualdad existente en la distribución del trabajo entre hombres y mujeres y también entre grupos de personas diferentes, como la diferencia en tipos, tiempos y valorizaciones de trabajos entre el sector urbano y rural. Además, los estudios sobre la precarización del trabajo de mujeres también arrojan luz sobre las maneras en que se constituyen desigualdades materiales y subjetivas necesarias para estructurar la redistribución desigual del riesgo. Finalmente, en el último apartado, hemos visto cómo el enfoque feminista ayuda entender nuevas formas producción de valor y su explotación desde lugares externalizados de la acumulación de capital.

Otro punto metodológico clave es la constante insistencia del feminismo en la necesidad de que las personas que experimentan desigualdades se 
constituyan en sujetos del conocimiento. Las poblaciones más vulnerables con sus actividades y prácticas sociales, culturales, reproductivas - se deben considerar fuentes de conocimiento para entender la configuración de las economías, los mercados y el Estado, analizando desde las realidades de estos grupos, desde su contexto, su experiencia y su situación identitaria (Vásconez 2012). Además de los aportes conceptuales y metodológicos de la economía feminista, tal vez la más importante contribución de la economía feminista al estudio de la economía viene en este sentido epistemológico: nos exige reconocer la politicidad de nuestras categorías conceptuales y de nuestros análisis del capital.

\section{Referencias bibliográficas}

Anderson, Jeanine (2007), "Género de cuidados". En Fronteras interiores: identidad, diferencia y protagonismo de las mujeres. Lima: Instituto de Estudios Peruanos.

Bermúdez, Héctor (2015), Venta directa por catálogo en Cali: hacia una conceptualización del trabajo. Tesis para obtener el título de maestría. Ecuador: FLACSO.

Boltanski Luc y Evé Chiapello (2002), El nuevo espíritu del capitalismo. Madrid: Ed Akal.

Brenner, Robert (2009) "What is Good for Goldman Sachs is Good for America: The Origins of the Present Crisis". Los Angeles: Center for Social Theory and Comparative History. http://escholarship.org/uc/item/0sg0782h. Carrión, Diego y Stalin Herrera (2012), Ecuador rural del siglo XXI. Soberanía alimentaria, inversión pública y politica agraria. Quito: Instituto de Estudios ecuatorianos.

Carrasco, Cristina (2003), "La sostenibilidad de la vida humana: ¿un asunto de mujeres?". Mujeres y trabajo: cambios impostergables. Magdalena León T. (Comp). Brasil: CLACSO, REMTE, ALAI y Marcha Mundial de las Mujeres: 11-49.

Christophers, Brett (2015) "Value models: Finance, risk, and political economy". Finance and Society 1.2:1-22. 
Dalla Costa, María Rosa y Selma James (1971), Las mujeres y la subversión de la comunidad. México: Siglo XXI.

Del Río, Sara y Amaia Pérez O. (2002), “La economía desde el feminismo: trabajos y cuidados”, en Rescoldos, Revista de diálogo social, 7: 15-36.

Federici, Silvia (2004), Calibán y la bruja. Mujeres, cuerpo y acumulación originaria. Madrid: Traficantes de Sueños.

(2013), Revolución en punto cero: trabajo doméstico, reproducción y luchas feministas. Madrid: Traficantes de Sueños.

Fumagalli, Andrea (2010), Bioeconomía y Capitalismo Cognitivo. Madrid: Traficantes de Sueños.

Gago, Verónica (2014) Razón Neoliberal: Economias barrocas y pragmática popular. Buenos Aires: Tinta Limón.

Galcerán, Monserrat (2006), “Introducción: producción y reproducción en Marx", en Transformaciones del trabajo desde una perspectiva feminista. Producción, reproducción, deseo, consumo, 13-26, Madrid: Laboratorio feminista.

Haraway, Donna (1995) Ciencia, cyborgs y mujeres: la reinvención de la naturaleza. Valencia: Universitat de València.

Harvey, David (2004), El nuevo imperialismo. Madrid: Ediciones Akal. (2008) La condición de la posmodernidad. Buenos Aires:

Amorrortu.

Hardt, Michael y Antonio Negri (2005), Imperio. Barcelona, México, Buenos Aires: Paidós.

(2004) Multitude: War and Democracy in the Age of Empire. Cambridge: Harvard University.

revolución del común. Madrid: Ed Akal.

(2011) Commonwealth. El proyecto de una

Himmelweit, Susan (2011), "El descubrimiento del 'trabajo no remunerado": consecuencias sociales de la expansión del término 'trabajo'. Trabajo de cuidado. Historia, teoría y políticas. C. Carrasco, C. Borderías y T. Torns 
(eds.). Madrid: Catarata,: 199 -224.

Hochschild, Arlie (1983), The managed heart. Commercialization of human feeling. Los Angeles: University of California Press.

Lipuma, Edward y Lee, Benjamin. 2004. Financial derivatives and the globalization of risk. Durham, NC: Duke University Press.

Marazzi, C. (2015), "Money \& Financial Capital”. Theory, Culture \& Society 32.7-8: 29-50.

Marx, Karl (1993), Grundrisse. New York: Penguin, 1993.

Míguez, Pablo (2008), "Las transformaciones recientes de los procesos de trabajo: desde la automatización hasta la revolución informática." Trabajo y Sociedad. Indagaciones sobre el trabajo, la cultura y las prácticas politicas en sociedades segmentadas $\mathrm{N}^{\circ} 11$.

Miyata, Hideko, y Julio Suzuki (2011), Trabalho, redes e territórios nos circuitos da economia urbana: uma análise da venda direta em Jundiai e Regiào Metropolitana de Sao Paulo. Faculdade de Filosofia, Letras e Ciências Humanas da Universidade de São Paulo.

Morini, Cristina (2014), Por amor o a la fuerza. Feminización del trabajo y biopolítica del cuerpo. Madrid: Traficantes de Sueños.

Negri, Antonio y Maurizio Lazzarato, M. (2001): Trabajo Inmaterial. Formas de vida y producción de subjetividad, DP\&A Editora, Río de Janeiro.

Pérez Orozco, Amaia (2014), La subversión feminista de la economia. Aportes para un debate sobre el conflicto capital-vida. Madrid: Traficantes de Sueños.

Picchio, Antonella (1994), "El trabajo de reproducción, tema central en el análisis del mercado de trabajo". Las mujeres y el trabajo. Rupturas conceptuales.

C. Borderías, C. Carrasco y C. Alemany (Comp.). Barcelona y Madrid: IcariaFUHEM: 453-490.

Quijano, Anibal (2000), Colonialidad del poder, eurocentrismo y Am Lat. Lima: CIES. 
Quiroga, Natalia y Diana Gómez Diana (2013), “QQué tiene para aportar una economía feminista decolonial a las otras economías?” Publicado en Agencia Latinoamericana de Información $8 \mathrm{feb}$. http://www.alainet.org/es/ active/61512. Consultado 28 enero 2016.

Salas, Mora y Pérez Sáinz (2006), "De la vulnerabilidad social al riesgo de empobrecimiento de los sectores medios”. Estudios sociológicos: 99-138.

Scott, Joan W (1993), "La mujer trabajadora en el siglo XIX". Historia de las mujeres. El siglo XIX. Tomo IV. G. Duby y M. Perrot (eds.). Madrid: Taurus: $405-435$.

Scott, Joan y Louise Tilly (1978) Women, work and family. NY: Holt, Rinehart \& Winston.

Terranova, Tiziana (2015), "Introduction to Eurocrisis, Neoliberalism and the Common”. Theory, Culture \& Society 32.7-8: 5-23.

Todaro, Rosalba y Sonia Yáñez (2004), El trabajo se transforma. Relaciones de producción y relaciones de género. Chile: Centro de Estudios de la Mujer.

Vásconez, Alison (2012), La Economia Feminista desde América Latina. Una hoja de ruta sobre los debates actuales en la región. Eds. Molano Adriana,

Elisabeth Robert y Anell Abreu. República Dominicana: ONU Mujeres.

Vega, Cristina (2015), "Dilemmas of Paid Home-care for the Elderly in Spain: Daughters, Elderly and Domestic Employees". Employers, Agencies and Immigration. London: Ashgate: 73-93.

Vercellone, Carlos (2013), "Re-leer la economía del conocimiento desde el antagonismo capital-trabajo". Publicado en Tesis11. http://www.tesis11. org.ar/capitalismo-cognitivo-releer-la-economia-del-conocimiento-desdeel-antagonismo-capital-trabajo/. 